Research Article

\title{
Risk Evolution of Ship Oil Spill in the Three Gorges Reservoir Area
}

\author{
Jie Yin and Ting Xiong (iD \\ Wuhan University of Technology, Wuhan, Hubei, China \\ Correspondence should be addressed to Ting Xiong; bearmos@163.com
}

Received 14 April 2021; Revised 13 June 2021; Accepted 5 July 2021; Published 15 July 2021

Academic Editor: Aditya Rio Prabowo

Copyright ( $\odot 2021$ Jie Yin and Ting Xiong. This is an open access article distributed under the Creative Commons Attribution License, which permits unrestricted use, distribution, and reproduction in any medium, provided the original work is properly cited.

\begin{abstract}
Ship oil spill accident will lead to enormous hazards in the Three Gorges Reservoir area. The risk mitigation of the ship oil spill is significant to the stakeholder of the maritime safety administration. To study the risk evolution of the ship oil spill in the Three Gorges Reservoir area, the framework of ship oil spill risk evolution was proposed according to the general definition of the risk; then, risk evolutions were analyzed from the perspectives of expected probability, system constraints of accident developing, and possible harmful consequences based on accidents analyses, simulation experiments, and structural equation model analysis. The results indicate that the system constraints of accident developing, such as the quantity of oil spills, free evolution time, and wind and flow conditions, have significant impacts on risk evolution of the ship oil spill after the occurrence of an accident in the Three Gorges Reservoir area.
\end{abstract}

\section{Introduction}

1.1. Research Background. Oil spill accident is one of the most typical and serious environmental pollution accidents in the process of human use of oceans and rivers. In recent years, oil spill accidents such as the "Prestige" oil spill, Dalian Xingang " 7.16 " oil pipeline explosion, Penglai "19-3" oilfield oil spill, and BP oil spill in the Gulf of Mexico had seriously affected the local and global aquatic environment, which further highlighted the importance and urgency of enhancing oil spill emergency response capability worldwide. With the rapid development of national economy, a steady growth is shown in China's demand for petroleum energy, number of related water transportation activities, as well as the risk of oil spill accidents.

The Three Gorges reservoir area of the Yangtze River is a special navigable water area with high social concern and high relevance to people's livelihood. Its navigation safety, cleanliness, and smoothness are closely related to the economic and social development along the river. In recent years, transportation of dangerous goods by water in the Three Gorges reservoir area has been rapidly increasing. Meanwhile, a considerable number of ships with irregular oil-containing sewage operations also increase the risk of oil spill accidents. According to the statistics of the Yangtze River Maritime Safety Administration, more than 100 oil spill accidents have occurred in the area in the past 20 years. In the Three Gorges reservoir area, many environmental pollution sensitive resources and unique hydrological conditions exist. Once an oil spill accident occurs, it will directly harm or damage the water environment and the riverside economies and may also indirectly cause adverse social impact. So, it is of great significance to enhance the emergency response capability and minimize the oil pollution risk in the Three Gorges reservoir area.

\subsection{Literature Review}

1.2.1. Ship Oil Spill Hazard. In terms of vessel-related oil spills, Murray studied the diffusion mechanism of offshore marine oil pollution and showed emergency responders how to formulate a ship oil pollution treatment scheme [1]. Kerkhoff et al. studied the surface distance, velocity, and content of deepwater oil spill weathering [2]. In terms of the evaluation oil damage, Geng summarized eight factors affecting the location of the oil spill and established a mathematical model to evaluate the level of oil spill accident 
by the fuzzy neural network [3]. Wu proposed that the level of oil spill accidents can be evaluated by factors such as oil spill, oil characteristics, meteorological conditions, and environmental sensitivity of the accident area [4]. This evaluation method reduces the influence of human factors when the indicators are quantified and proposes to refer to the spill. The results of the oil simulation and oil spill cleanup simulations determine the threat level of the oil spill and the level of the oil spill. In terms of environmental and economic damage caused by oil spills on ships, Pan et al. studied the economic losses caused by the Penglai 19-3 oilfield drainage incident in the northeastern China's Bohai Sea waters to the Yantai fishery and estimated the economic loss of the oil spill from the Yantai fishery and related marine aquaculture. The estimated economic loss is at least 1.256 billion yuan [5].

1.2.2. Ship Oil Spill Risk Assessment. Devnaney and Stewart used Bayesian analysis to conduct preliminary research on the probability characteristics of oil spill accidents and oil spills, based on historical and unofficial statistics and onsite survey data. Liu et al. established a ship and platform oil spill risk probability function model, and based on the historical data from 1973 to 2002, they analyzed the oil spills in Bohai Bay ships and platforms [8]. In the research on ship oil spill risk assessment, Li and Huang used fuzzy comprehensive evaluation to conduct oil spill risk zoning, combined with the port environmental sensitivity map for risk impact assessment, and provided scientific basis for the port oil spill contingency plan [9]. Zhang and Thai based on brainstorming and Bayesian networks to study the risk assessment of ship oil spills under information uncertainty $[10,11]$.

1.2.3. Ship Oil Spill Prevention and Control Management. Blindow et al. introduced two models of pollution in Tåkern and Krankesjön Lake and proposed pollution prevention and control measures for freshwater lakes [12]. Li [13-15] conducted research on the status of ship pollution in the Three Gorges reservoir area, pollution hazard, prevention and control measures, risk prediction, emergency response, etc. The risk assessment and precontrol of the accident were carried out, and countermeasures against pollution accidents were proposed. Singh et al. studied the oil spill risk prevention and control management system in the Caribbean and pointed out that the prevention and control management system consisting of supervision, standards, monitoring procedures, data collection, and more effective conventions can be effective to reduce the risk of oil spills caused by ships in the Caribbean [16].

In summary, existing works mainly focus on ship oil spill accident rating, ship oil spill damage to the ecological environment and causing losses, ship oil spill risk assessment, oil spill hazard prediction, and oil spill prevention and control management system development. There are few studies on the risk evolution of ships' oil spill in a large-scale inland reservoir area.
1.3. Aim and Innovation. From the important gaps in the literatures and the demand of emergency response decisionmaking of ship oil spill accidents, this paper studies the risk evolution of the ship oil spill in the Three Gorges Reservoir area. The innovation of this study is twofold. The first one is proposing the framework of ship oil spill risk evolution from the perspectives of expected probability, system constraints, and harmful consequence. The second one is analyzing risk evolutions of the ship oil spill in the Three Gorges Reservoir area from the perspectives of expected probability, system constraints of accident developing, and possible harmful consequences.

\section{Ship Oil Pollution and Prevention in the Three Gorges Reservoir Area}

2.1. Ship Oil Spill Accidents in the Area. Dangerous goods transported by waterway in the Three Gorges Reservoir area mainly include bulk chemical tankers, petroleum products, and packaging dangerous goods. Most of the pollution accidents are sudden accidents. From 1997 to 2018, there were 549 water traffic accidents in the Three Gorges reservoir area. The types of ships with major pollution accidents in the Three Gorges reservoir area are mainly oil tankers and chemical tankers. Meanwhile, passenger ships and general cargo ships also have multiple pollution accidents, but the amount of pollutants leaked is relatively small. The number of oil spill accidents in the Three Gorges reservoir area shows the downward trend from 1997 to 2018 resulting from strict maritime supervision on transport ship and risk mitigation of the ship oil spill. The details of 142 ship oil spill accidents from 1997 to 2018 are shown in Table 1.

2.2. Oil Spill Sensitive Areas. According to statistics, there are 67 water intakes (more than 50 living water intakes), 1 aquaculture farm, and more than 20 provincial nature reserves along the coast of the Three Gorges reservoir area; there are 20 scenic zones, 300 scenic spots, 4 national key scenic spots, 7 national key cultural relics protection units, and 2000 cultural relics protection units and cultural relics. The distribution of main environmentally sensitive resources (circled) in the Three Gorges Reservoir area is shown in Figure 1. The environmentally sensitive resources could all be polluted by the oily water resulting from ship oil spill accidents. If the environmentally sensitive resources are polluted by the oily water, there will be huge harmful consequences, which includes the loss of public safety, environmental pollution, cleaning costs, and damage of economics.

\section{Framework of Ship Oil Spill Risk Evolution}

3.1. Framework of Ship Oil Spill Risk Assessment. According to the general definition of the risk, the risk of a ship oil spill is the function of the expected probability of a ship oil spill accident, the system constraints of accident developing, and the possible harmful consequences of the accident under the system constraints $[17,18]$, which are shown in Figure 2. 
TABLE 1: Number of oil spill accidents in the area from 1997 to 2018.

\begin{tabular}{lccccccccccc}
\hline Year & 97 & 98 & 99 & 00 & 01 & 02 & 03 & 04 & 05 & 06 & 07 \\
\# of accidents & 11 & 14 & 22 & 10 & 9 & 17 & 8 & 6 & 4 & 8 \\
\hline Year & 08 & 09 & 10 & 11 & 12 & 13 & 14 & 15 & 16 & 17 \\
\# of accidents & 5 & 6 & 3 & 2 & 3 & 1 & 2 & 2 & 2 & 0 \\
\hline
\end{tabular}
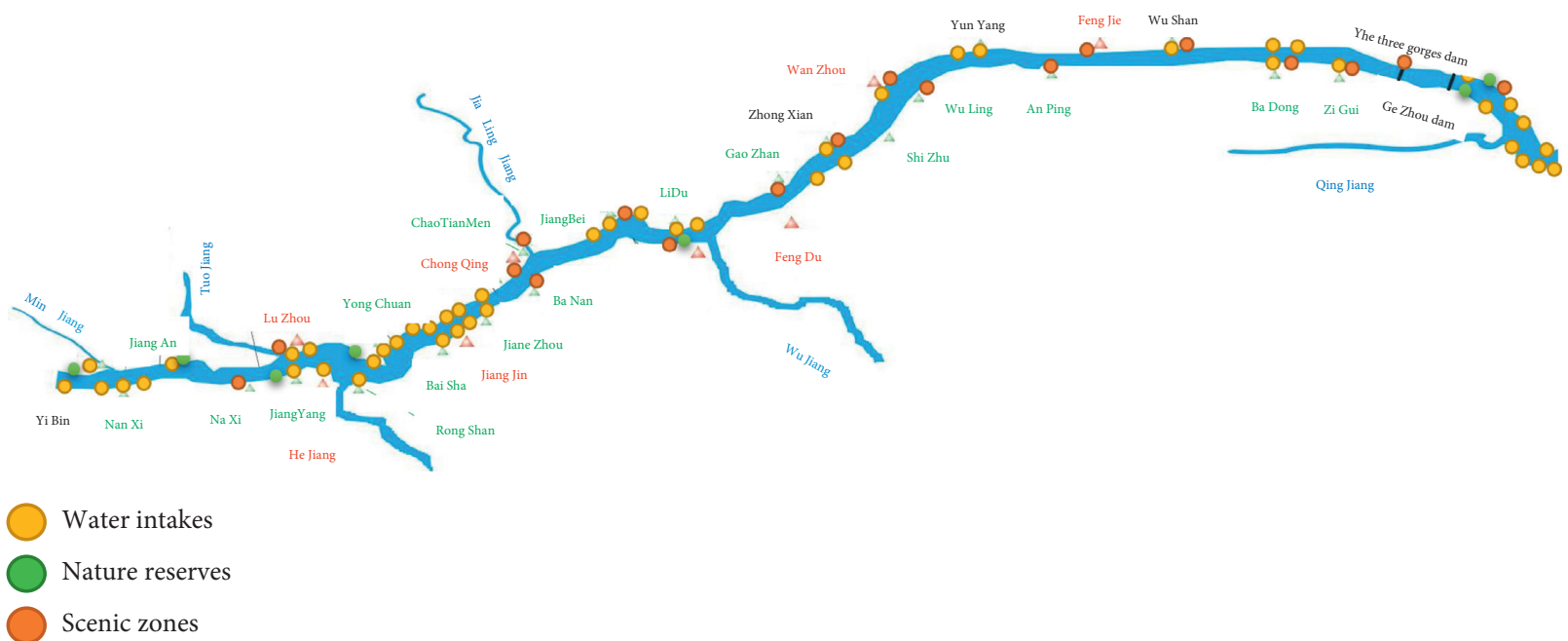

FIgURE 1: Schematic diagram of the distribution of main environmentally sensitive resources in the Three Gorges Reservoir area.

The expected probability of a ship oil spill accident is mainly related to the operational ship elements, the navigational environmental conditions, and the ship's class-level standards. The operational ship elements mainly refer to the type of ship, the age of the ship, the tonnage of the ship, etc. The navigational environmental conditions mainly refer to the parameters of navigation channel, navigation density, wind, water flow, visibility, etc. The class-level standards mainly refer to the airworthiness of the ship, the skill level of the crew, etc.

The system constraints of accident developing mainly refer to the amount of oil spill, free evolution time, and form of diffusion drift.

The hazards caused by oil spill accidents in the Three Gorges reservoir area mainly refer to the loss of public safety, environmental pollution, cleaning costs, and damage of economics.

Combining the definition of ship oil spill risk and the above characteristics, the function of ship oil spill risk is proposed as follows:

$$
R=P \cdot f\left(z_{1}, z_{2}, z_{3}\right) \cdot S,
$$

where $R$ is the risk of oil spills from ships, $P$ is the expected probability of ship oil spill accidents related to ship elements, navigational environmental conditions, and classification standards, $f\left(z_{1}, z_{2}, z_{3}\right)$ are the coefficients of system constraints caused by oil spill accidents related to the quantity of oil spills $z_{1}$, free evolution time $z_{2}$, wind and flow conditions $z_{3}$, etc., the greater the coefficient of system constraints, the greater the hazard caused by oil spill accidents, and $S$ is the degree of damage caused by oil spill accidents, mainly the loss of public safety hazards, the loss of environmental pollution, the cost of clean-up costs, and the economic hazard losses.
3.2. Evolution Ways of Ship Oil Spill Risk. According to the general definition of ship oil spill risk, evolution ways of ship oil spill risk can be represented as follows.

(1) The greater the expected probability of a ship oil spill accident under the same conditions, the greater the risk of an oil spill

(2) The more unfavorable the system constraint conditions of the accident developing (the quantity of oil spills, free evolution time, wind and flow conditions, etc.), the greater the danger of oil spill accidents and the greater the risk of oil spill accidents

(3) Under the same conditions, the greater the damage caused by an oil spill, the greater the risk of an oil spill

\section{Risk Evolution Analysis of Ship Oil Spill in the Three Gorges Reservoir Area}

\subsection{Risk Evolution of Ship Oil Spill from the Perspective of Expected Probability}

4.1.1. Composition of Expected Probability of Ship Oil Spill Accident

(1) The Expected Probability of a Ship Oil Spill Accident. The expected probability of a ship oil spill accident is determined by the causal probability of the navigational environmental conditions, ship factors, and classification standards. The expected probability calculation function of a ship oil spill is shown in the following equation:

$$
P=f\left(X_{1}, X_{2}, X_{3}\right),
$$




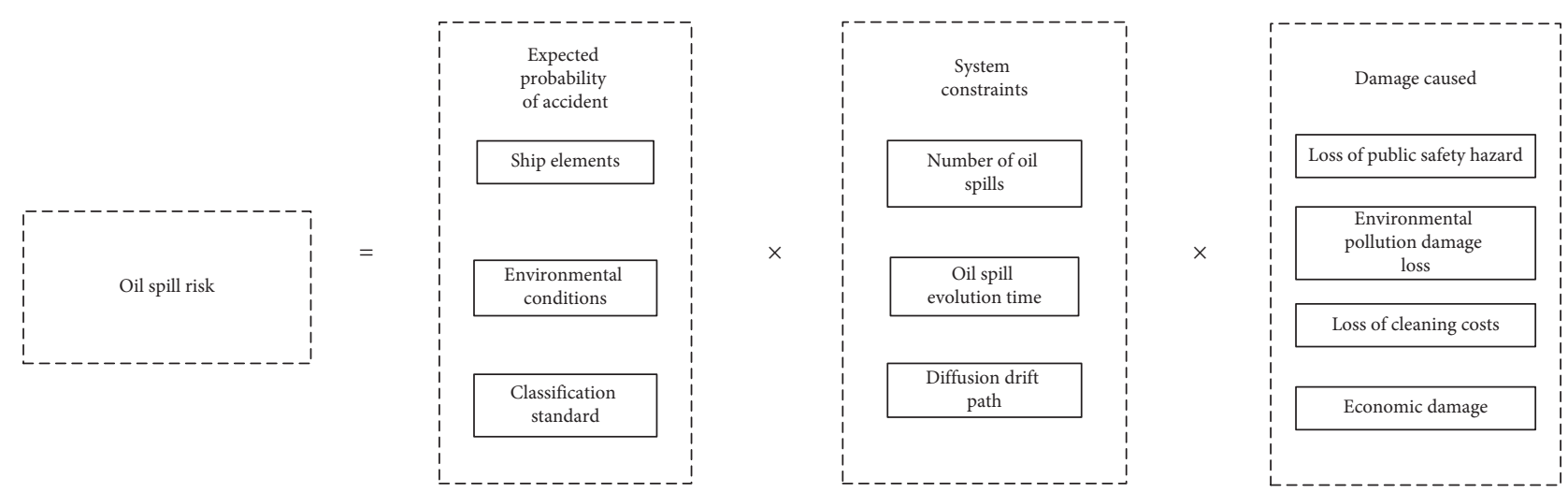

FIgURE 2: Risk of the oil spill accident.

where $P$ is the expected probability of a ship oil spill accident, $X_{1}$ is environmental conditions, $X_{2}$ is the ship factor, and $X_{3}$ is the classification standard.

$$
P=\left\{\begin{array}{l}
P_{X_{1}}\left(1+P_{X_{2}}+P_{X_{3}}\right) \subset(0,1], \\
P_{X_{2}}\left(1+P_{X_{1}}+P_{X_{3}}\right) \subset(0,1], \\
P_{X_{3}}\left(1+P_{X_{1}}+P_{X_{3}}\right) \subset(0,1],
\end{array}\right.
$$

where $P$ is the expected probability of ship oil spill accident, $P\left(X_{1}\right)$ is the probability of the oil spill due to ship factors, $P_{-}\left(X_{2}\right)$ is the probability of oil spill accident due to navigational environmental conditions, and $P\left(X_{3}\right)$ is the probability of oil spill accident due to classification standards.

First, compare the values of $P\left(X_{1}\right), P\left(X_{2}\right)$, and $P\left(X_{3}\right)$, and take the larger one as the main factor of the occurrence probability; the other two factors are the additional cause occurrence probability; the total occurrence probability is approximately equal to the main cause probability times the additional cause probability sum with 1 .

For example, if a ship 's oil spill accident has a high probability in a ship 's factors, navigation conditions, and classification standards under certain conditions, the ship 's oil spill accident probability is mainly considered in the ship 's factor; meanwhile, there are certain risks of navigation. The probability of oil spill accidents caused by conditions and class standard factors is higher than the probability of oil spill accidents caused by risk conditions and classification standard factors; therefore, the overall probability is approximately pollution probability caused by ship factors $\times(1+$ probability of pollution caused by sailing conditions + probability of pollution caused by classification standards.

(2) The Expected Probability of the Quantity of Oil Spilled. The expected probability of the quantity of oil spilled is determined by the type of oil spilled ship and the type of oil spill accident. Specifically, it is shown in the following formula:
According to the above physical meaning, from the perspective of step difference, we try to establish equation (3) to characterize the expected probability of an oil spill accident:

$$
\begin{aligned}
& \text { if } P_{X_{1}}=\max \left(P_{X_{1}}, P_{X_{2}}, P_{X_{3}}\right) \\
& \text { if } P_{X_{2}}=\max \left(P_{X_{1}}, P_{X_{2}}, P_{X_{3}}\right), \\
& \text { if } P_{X_{3}}=\max \left(P_{X_{1}}, P_{X_{2}}, P_{X_{3}}\right), \\
& \qquad P_{v}=f\left(Y_{1}, Y_{2}\right),
\end{aligned}
$$

where $P_{v}$ is the expected probability of the quantity of oil spilled, $Y_{1}$ is the type of oil spilled ship, and $Y_{2}$ is the type of oil spill accident.

According to assembly of the type of oil spilled ship and the type of oil spill accident, the details are shown in the following equation:

$$
P_{V}= \begin{cases}P_{Y_{1}=1}\left(1+P_{Y_{2}=1}\right), & \text { if } V \subset(0,100 \mathrm{~kg}], \\ P_{Y_{1}=1}\left(1+P_{Y_{2}=2}\right), & \text { if } V \subset(100 \mathrm{~kg}, 7 t], \\ P_{Y_{1}=2}\left(1+P_{Y_{2}=1}\right), & \text { if } V \subset(7 t, 700 t], \\ P_{Y_{1}=2}\left(1+P_{Y_{2}=2}\right), & \text { if } V \subset(700 t, 10000 t],\end{cases}
$$

where $P_{V}$ is the expected probability of the quantity of oil spilled from the ship; the quantity of oil spilled $V$ is quantified in four levels, and the value intervals are $(0$, $100 \mathrm{~kg}],(100 \mathrm{~kg}, 7 t],(7 t, 700 t]$, and $(700 t, 10000 t] . P\left(Y_{1}\right)=1$ is the expected probability of an oil spill in a nonoil tanker; $P$ $\left(Y_{1}\right)=2$ is the expected probability of an oil spill in an oil tanker; $P\left(Y_{2}\right)=1$ is the expected probability of the operational oil spill; $P\left(Y_{2}\right)=2$ is the expected probability of the accidental oil spill.

4.1.2. Evolution of Expected Probability of Ship Oil Spill. Based on the data of 142 ship oil spill accidents, the evolution rules of ship oil spill risk in the Three Gorges reservoir area were analyzed. 
In theory, there is no direct relationship between the expected probability of an accident and the accident hazard. In practice, due to strict oil pollution prevention management on ships and rivers, the society, government, shipping companies, and ships have adopted various oil pollution prevention and control measures to reduce the probability of oil spill accidents and the hazard of oil spill accidents. According to data of 142 ship oil spill accidents, the accidents with large oil spill hazards occur less frequently; the accidents with small oil spill hazards occur relatively frequently. The details are shown in Figure 3.

According to data of 142 ship oil spill accidents, the relations between the expected probability of the quantity of oil spilled and types of oil spilled ship and oil spill accident are shown in Figure 4.

\subsection{Risk Evolution of Ship Oil Spill from the Perspective of System Constraints of Accident Developing}

4.2.1. Composition of System Constraints of Accident Developing. After the spill, whether to lead to cause damage or the magnitude of the damage is related to several conditions. First of all, whether the oil spill is harmful is related to the cleanup intervention. Once the oil spill occurs on the ship, the relevant clean-up measures will be taken immediately, which will not lead to major damage; the magnitude of the oil spill is related to the timeliness of the clean-up intervention, and the damage will be small if the clean-up intervention is timely. Secondly, ship oil spill damage is related to the form and path of diffusion drift. Different form and path cause various results. The magnitude of the oil spill is also related to the amount of oil spill. Large quantities of oil spills have a high probability of causing high damage. Therefore, damage of the ship oil spill in the Three Gorges Reservoir area is related to the system constraints of accident developing, namely, the amount of the oil spill, the free evolution time of the oil spill, and the form of diffusion drift.

Amount of oil spill: the quantification of the quantity of oil spills of ships is divided into four grades, that is, it is divided into extraordinarily serious oil spills, serious oil spills, large oil spills, and ordinary oil spills.

Free evolution time: the quantification of the free evolution time of the ship's oil spill is divided into four levels, namely, the evolution of laissez-faire freedom, the long-term free evolution, the medium-term free evolution time, and the short-term free evolution time.

Diffusion drift form: the form and path of the oil spill diffusion in the reservoir area are mainly determined by the wind and flow conditions. The classification of the wind and flow conditions that restrict the ship's oil spill diffusion drift is classified into four levels, namely, severe wind-flow condition, unfavorable wind-flow condition, ordinary wind-flow condition, and less affected wind-flow condition.

4.2.2. Evolution of System Constraints of Accident Developing. According to data of simulation experiment and accidents' analyses data in the Three Gorges Reservoir area, the

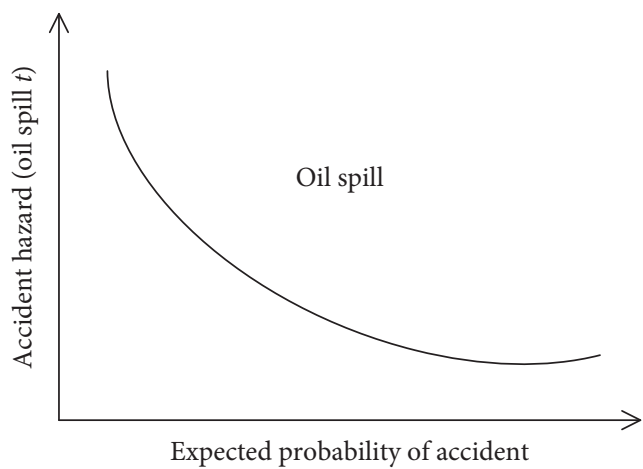

FIGURE 3: Relationship between the expected probability of an accident and the accident hazard.

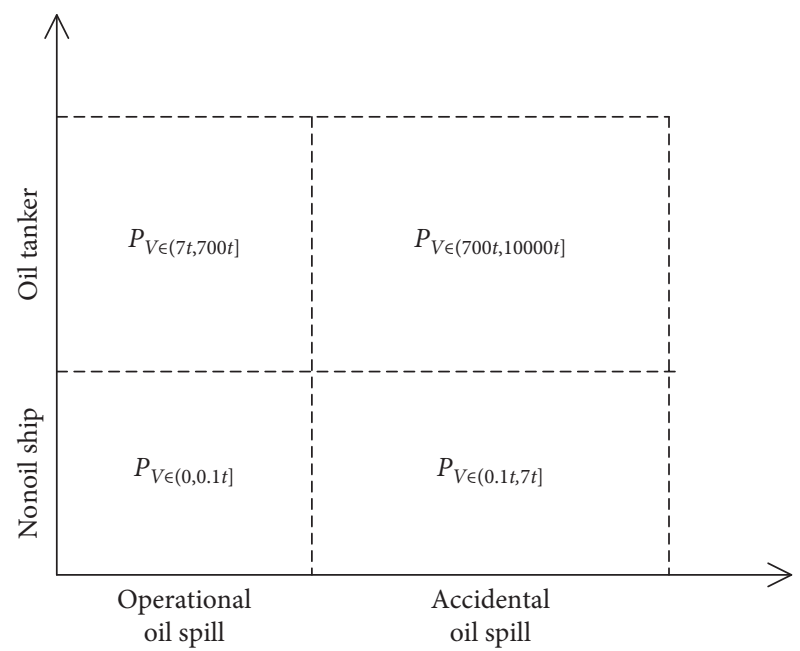

FIGURE 4: Relationship between the quantity of oil spill and its related elements.

greater the quantity of oil spills, the greater the hazard of oil spill accidents (environmental pollution hazards, loss of clean-up costs, and economic losses). Therefore, under the same conditions, the greater the quantity of the oil spill, the greater the risk of an oil spill accident. The details are shown in Figure 5.

According to data of the simulation experiment in the Three Gorges Reservoir area and other related research, oil spill diffusion, drift, and weathering are mainly concentrated during the 10 100 hours after the occurrence of a ship oil spill. During the period, the diffusion and drift behavior are the mainstay of oil spill hazard. While the period is also the key time for oil spill emergency response, after 100 hours, the spreading behavior gradually weakens, and the drift behavior evolves according to environmental conditions. Therefore, the change and duration of the oil spill in water can be shown in Figure 6 .

According to data of simulation experiment and accidents' analyses data in the Three Gorges Reservoir area, the longer the free evolution time, the greater the hazard of oil spill accidents (environmental pollution hazards, loss of pollution cleaning costs, and economic hazard losses). Therefore, under the same conditions, the areas where the oil-based pollution prevention shore power is scarce or the offshore base forces are far away from oil spills; the lower the probability of timely and effective intervention of oil spills, 


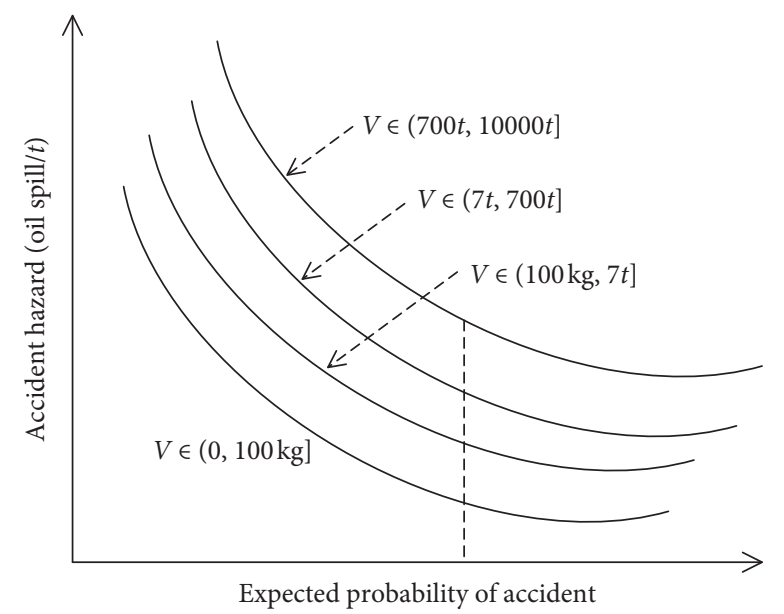

FIgURE 5: Relationship between oil spills' quantity and oil spill risk.

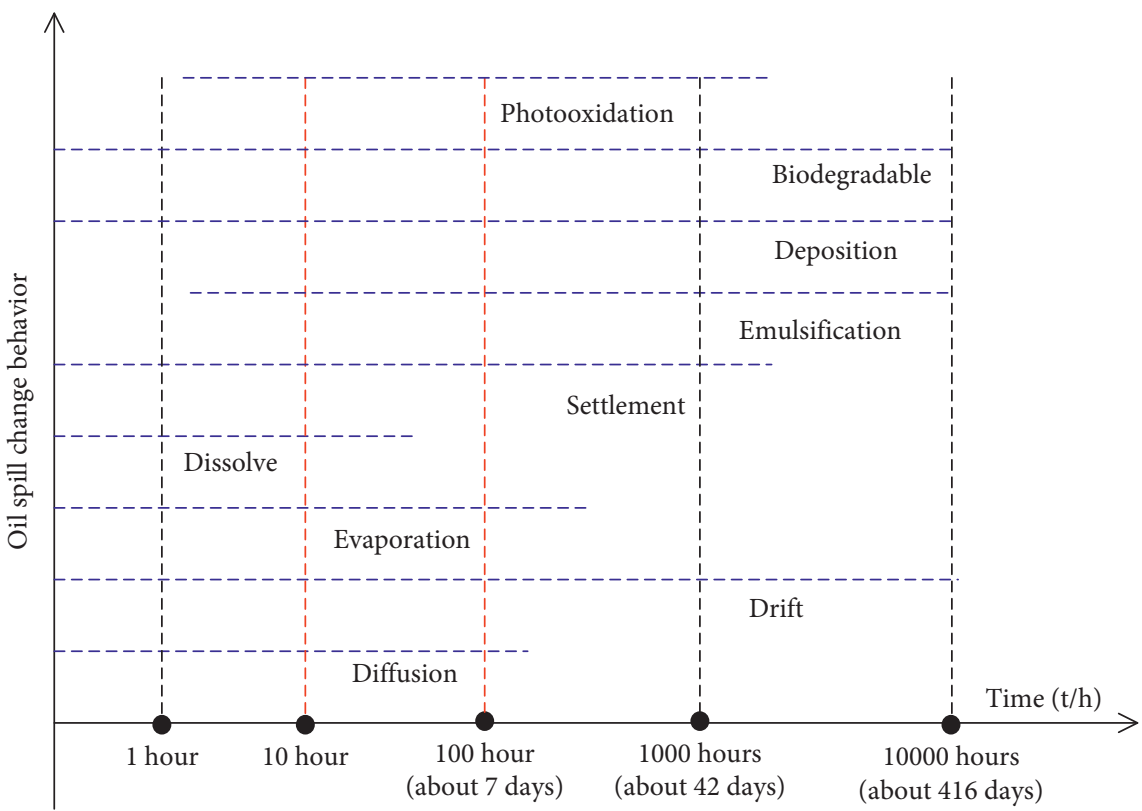

Figure 6: Change and duration of the oil spill in water.

the longer the free evolution time of oil spills, the greater the danger of an oil accident, and the greater the risk of an oil spill. The details are shown in Figure 7.

According to data of simulation experiment and accidents' analyses data in the Three Gorges Reservoir area, the worse the wind and flow conditions, the greater the expected probability of ship oil spill accidents, and the greater the damage of oil spill accidents (environmental pollution hazards, loss of cleanup costs, and economy damage). Therefore, under the same conditions, the worse the wind and flow conditions, the sharper the risk of oil spill accidents. The details are shown in Figure 8.

\subsection{Risk Evolution of Ship Oil Spill from the Perspective of Hazards Caused by Oil Spill Accidents}

4.3.1. Composition of Ship Oil Spill Hazards. The ship oil spill hazards in the Three Gorges reservoir area mainly refer to the loss of public safety, environmental pollution, cleaning costs, and damage of economics.

Public safety hazard loss: public safety hazards are classified into four levels by level quantification, which is divided into major malignant event losses, major public safety hazard losses, medium safety hazard losses, and minor safety incident losses 


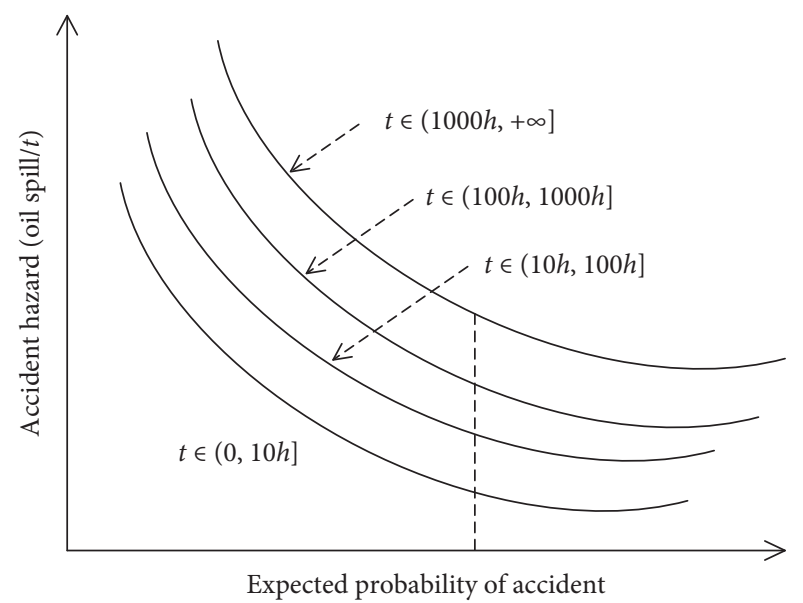

FIGURE 7: Relationship between oil spills' quantity and oil spill risk.

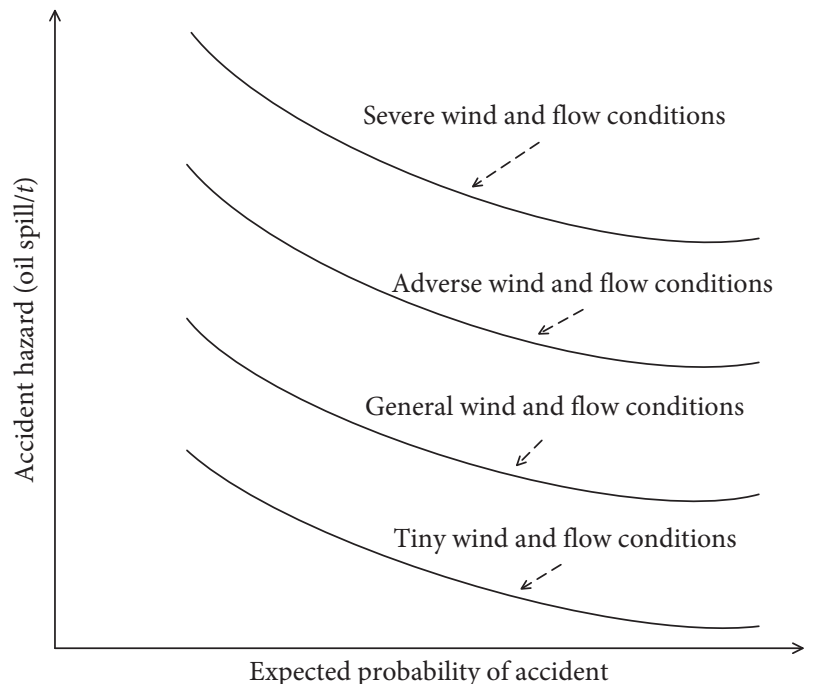

Figure 8: Relationship between wind and flow conditions and accident risk.

Environmental pollution damage loss: the loss of environmental pollution hazard is divided into four levels through grade quantification, which is divided into malignant pollution, serious pollution, moderate pollution, and mild pollution

Cleaning disposal cost loss: the environmental pollution hazard loss is divided into four levels through grade quantification, that is, it is divided into huge decontamination disposal cost loss, major decontamination disposal cost loss, medium decontamination disposal cost loss, and small decontamination disposal cost loss

Economic damage: the loss of economic hazard is divided into four levels by level quantification, which is divided into damage loss of huge economy, damage of major economic hazard, loss of hazard of medium economy, and damage of small economy

\subsubsection{Evolution of Ship Oil Spill Hazards Based on Structural Equation Model}

(1) Construction of Structural Equation Model. For the relationship between the oil spill damage and system constraints of accident developing, a structural equation path diagram can be established, as shown in Figure 9.

The structural equation is

$$
\left(\begin{array}{l}
\eta_{1} \\
\eta_{2}
\end{array}\right)=\left(\begin{array}{cc}
0 & 0 \\
\beta_{21} & 0
\end{array}\right)\left(\begin{array}{l}
\eta_{1} \\
\eta_{2}
\end{array}\right)+\left(\begin{array}{ll}
\gamma_{11} & \gamma_{21} \\
\gamma_{12} & \gamma_{22}
\end{array}\right)\left(\begin{array}{l}
\xi_{1} \\
\xi_{2}
\end{array}\right)+\left(\begin{array}{l}
\zeta_{1} \\
\zeta_{2}
\end{array}\right) \text {. }
$$

The measurement equation for the dependent variable is

$$
\left(\begin{array}{ll}
y_{11} & y_{12} \\
y_{21} & y_{22}
\end{array}\right)=\left(\begin{array}{ll}
\lambda_{11} & \lambda_{12} \\
\lambda_{21} & \lambda_{22}
\end{array}\right)\left(\begin{array}{l}
\eta_{1} \\
\eta_{2}
\end{array}\right)+\left(\begin{array}{ll}
\varepsilon_{11} & \varepsilon_{12} \\
\varepsilon_{21} & \varepsilon_{22}
\end{array}\right)
$$

where $\lambda_{11}, \lambda_{21}, \lambda_{12}$, and $\lambda_{22}$ are load terms and $\varepsilon_{11}, \varepsilon_{21}, \varepsilon_{12}$, and $\varepsilon_{22}$ are error terms. is

The measurement equation of the independent variable

$$
\left(\begin{array}{l}
x_{1} \\
x_{2} \\
x_{3}
\end{array}\right)=\left(\begin{array}{ll}
\lambda_{1} & 0 \\
0 & \lambda_{2} \\
0 & \lambda_{3}
\end{array}\right)\left(\begin{array}{l}
\xi_{1} \\
\xi_{2}
\end{array}\right)+\left(\begin{array}{l}
\delta_{1} \\
\delta_{2} \\
\delta_{3}
\end{array}\right),
$$

where $\lambda_{1}, \lambda_{2}$, and $\lambda_{3}$ are load terms and $\delta_{1}, \delta_{2}$, and $\delta_{3}$ are error terms.

(2) Model Calculation and Result Analysis. The model is solved by the iterative-based partial least squares algorithm. Based on 80 sets of ship oil spill simulation data with complete oil spill conditions (oil spill amount, free drift time, and diffusion drift form), the result of the model is obtained by the 19 iterative operations: $\gamma_{11}=0.7436, \gamma_{12}=0.8727, \gamma_{21}=0.6276$, and $\gamma_{22}=0.8213$

The amount of oil spill, free evolution time, and wind and flow conditions have a strong impact on indirect hazard losses (loss of cleaning costs and economic hazards); the amount of oil spills, free evolution time, and wind and flow conditions also have a strong impact on direct damage losses (public safety hazard losses) and environmental pollution losses.

The influence of the observed variables affecting the direct hazard loss indicators from large to small is oil spill quantity $>$ free evolution time $>$ wind and flow conditions; the influence of the observed factors affecting the indirect hazard loss indicators from large to small in order: oil spill quantity $>$ wind and flow conditions $>$ free evolution time.

The amount of the oil spill, free evolution time, and wind and flow conditions are the indicators of the factors affecting the evolution of oil spill hazard. In the case of a certain amount of oil spill, the free evolution time and wind and flow conditions have a greater impact on the evolution of oil spill hazards. 


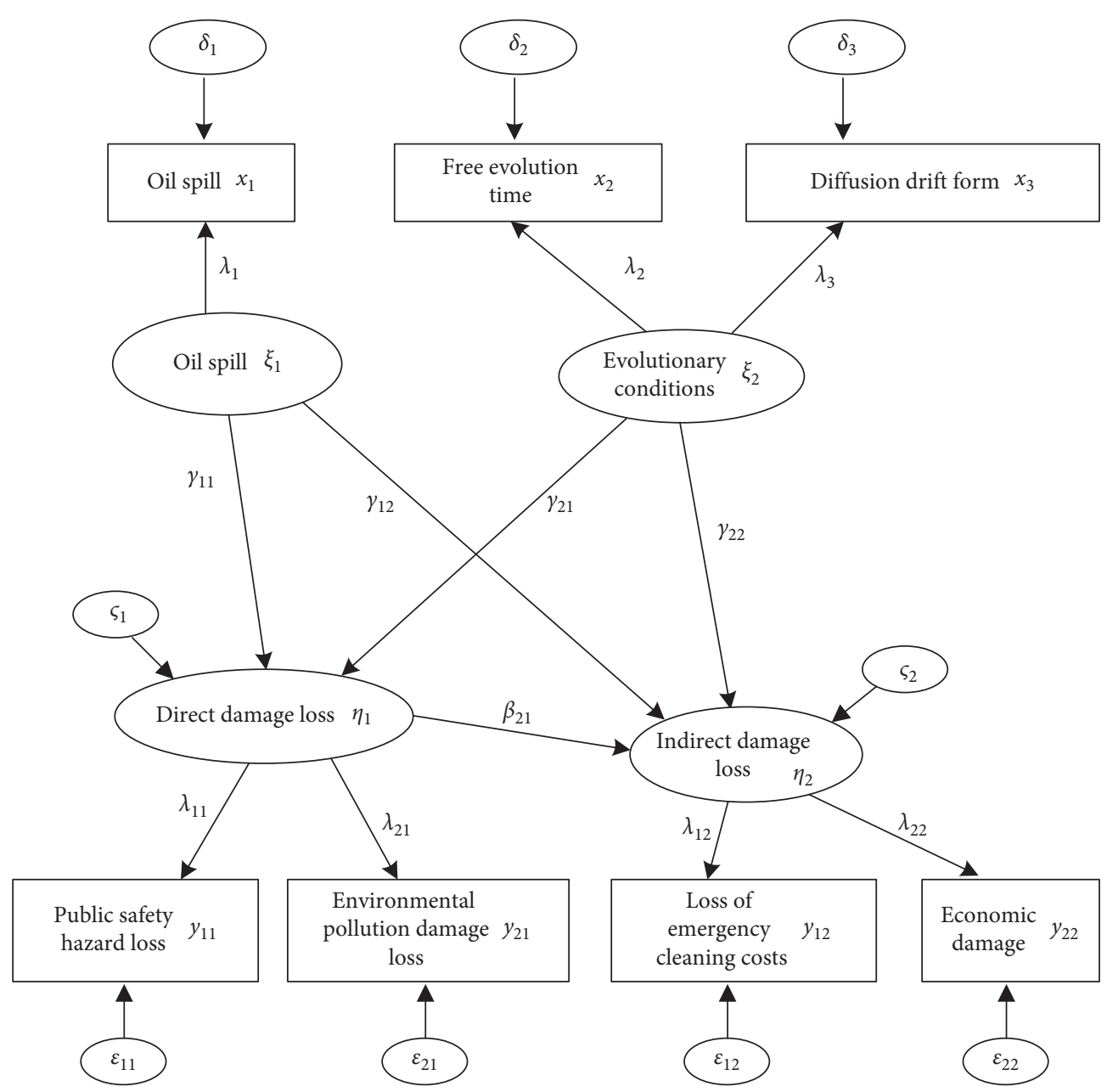

FIGURE 9: Structural equation path diagram for the relationship between development and hazard of the ship oil spill.

The observation variables of oil spill hazard loss in the measurement of oil spill hazard loss are in the descending order: loss of cleaning cost $>$ loss of economic hazard $>$ loss of environmental pollution hazard $>$ loss of public hazard.

The amount of the oil spill is more direct than the evolutionary condition factor on direct damage loss and indirect damage loss; the impact of oil spill on indirect hazard loss is greater than that on direct hazard loss.

Evolving conditional elements (free evolution time and spread drift form) have a strong impact on indirect hazard losses and have a greater impact on direct hazard losses. There is a certain impact between direct damage loss and indirect damage loss, but the impact relationship is not very strong.

4.4. Results. In conclusion, oil tanker or not, type of ship oil spill accidents, quantity of oil spills, free evolution time, wind and flow conditions, and composition of harmful consequences of oil spill accident have considerable impacts on risk evolution of the ship oil spill. Furthermore, the system constraints of accident developing, such as the quantity of oil spills, free evolution time, and wind and flow conditions, have significant impacts on risk evolution of the ship oil spill after the occurrence of an accident in the Three Gorges Reservoir area.

\section{Conclusions and Prospects}

5.1. Conclusion. To study the risk evolution of the ship oil spill in the Three Gorges Reservoir area, the framework of ship oil spill risk evolution was proposed; then, risk evolutions were analyzed from the perspectives of expected probability, system constraints of accident developing, and possible harmful consequences based on accidents analyses, simulation experiments, and structural equation model analysis.

The results indicate that the system constraints of accident developing, such as the quantity of oil spills, free evolution time, and wind and flow conditions, have significant impacts on risk evolution of the ship oil spill after the occurrence of an accident in the Three Gorges Reservoir area. The risk evolution rules of the ship oil spill could be used for decision-making of emergency response of ship oil spill accidents in the Three Gorges Reservoir area.

5.2. Future Works. Due to various cargoes and ships, changeful navigation environments, and composition of harmful consequences, the risk evolution of ship oil spills in the Three Gorges reservoir area is actually a very 
complicated system. More accidents' data and simulation experiment data should be used to analyse and verify risk evolution rules of the ship oil spill in the Three Gorges Reservoir Area.

\section{Data Availability}

The data used to support the findings of this study may be released upon application to the China Three Gorges Corporation: https://www.ctg.com.cn/.

\section{Conflicts of Interest}

The authors declare that they have no conflicts of interest.

\section{References}

[1] S. P. Murray, "Turbulent diffusion of oil in the ocean1," Limnology and Oceanography, vol. 17, no. 5, pp. 651-660, 1972.

[2] M. Kerkhoff, P. Frintrop, and W. Koops, "Pollution of the Dutch coast by oil from the "Eline V"-July 1978," Science of the Total Environment, vol. 19, no. 1, pp. 33-39, 1981, (in Chinese).

[3] X. Geng, Evaluation of Oil Spill Accident Threaten Degree \& Emergency Reaction Decision, Dalian Maritime University, Dalian, China, (in Chinese), 2000.

[4] Y. Wu, "Inland vessel oil spill degree evaluation method," China Water Transport, vol. 9, no. 6, pp. 12-13, 2009, (in Chinese).

[5] G. Pan, S. Qiu, X. Liu, and X. Hu, "Estimating the economic damages from the Penglai 19-3 oil spill to the Yantai fisheries in the Bohai sea of northeast China," Marine Policy, vol. 62, pp. 18-24, 2015.

[6] J. W. Devanney and R. J. Stewart, "Analysis of oil spill statistics," Primary, Physical Impacts of Offshore Petroleum Developments, vol. 126, p. 126, 1974.

[7] J. W. Devanney and R. J. Stewart, "Bayesian analysis of oil spill statistics," Marine Technology and SNAME News, vol. 11, no. 4, pp. 365-382, 1974.

[8] X. Liu, R. Meng, Q. Xing, M. Lou, H. Chao, and L. Bing, "Assessing oil spill risk in the Chinese Bohai sea: a case study for both ship and platform related oil spills," Ocean \& Coastal Management, vol. 108, pp. 140-146, 2015.

[9] P. Li and J. Huang, "Application of fuzzy comprehensive assessment to the division of oil spill risk from tankers in ports," Environmental Protection in Transportation, vol. 20, no. 2, pp. 12-14, 1999, (in Chinese).

[10] G. Zhang and V. V. Thai, "Expert elicitation and Bayesian network modeling for shipping accidents: a literature review," Safety Science, vol. 87, pp. 53-62, 2016.

[11] X. Hou, B. Hodges, D. Feng, and Q. Liu, "Uncertainty quantification and reliability assessment in operational oil spill forecast modeling system," Marine Pollution Bulletin, vol. 116, no. 1-2, pp. 420-433, 2017.

[12] I. Blindow, G. Andersson, A. Haregy, and S. Johansson, "Long-term pattern of alternative stable states in two shallow EUT rophic lakes," Freshwater Biology, vol. 30, no. 1, pp. 159-167, 1993.

[13] C. Li, "Current situation and prevention of ship pollution in three gorges reservoir area," Environmental Protection in Transportation, vol. 24, no. s1, pp. 71-73, 2003, (in Chinese).
[14] M. Huang, W. Wang, and Z. Tan, "Study on pollution in three gorges reservoir area," China Water Transport, vol. 8, pp. 177-178, 2004, (in Chinese).

[15] C. Xu, W. Shu, J. Cao, and Y. Wang, "Eutrophication and its harm forecast and prevention in water level-fluctuating zone of three gorges reservoir area," Resources and Environment in the Yangtze Basin, vol. 4, pp. 440-444, 2005, (in Chinese).

[16] A. Singh, H. Asmath, C. L. Chee, and J. Darsan, "Potential oil spill risk from shipping and the implications for management in the Caribbean sea," Marine Pollution Bulletin, vol. 93, no. 12, pp. 217-227, 2015.

[17] H. P. Li, Hierarchical Risk Assessment of Water Supply Systems, Loughborough University, Loughborough, UK, 2007.

[18] Z. Y. Cheng, P. Y. Wang, C. Y. Yang, L. F. Han, and S. X. Wang, "Method of three-dimensional measurement of maritime risk caused by landslide," Port \& Waterway Engineering, vol. 9, pp. 64-69, 2017. 